\title{
Large-Scalable Graphene Oxide Films with Resistive Switching for Non-Volatile Memory Applications
}

\author{
M. Brzhezinskaya ${ }^{\mathrm{a}, *}$, O.O. Kapitanova ${ }^{\mathrm{b}}$, O.V. Kononenko ${ }^{\mathrm{c}}, \mathrm{S}$. Koveshnikov ${ }^{\mathrm{c}}$, V. Korepanov ${ }^{\mathrm{c}}$, \\ D. Roshchupkin ${ }^{\mathrm{c}, * *}$
}

\begin{abstract}
${ }^{\text {a } H e l m h o l t z-Z e n t r u m ~ B e r l i n ~ f u ̈ r ~ M a t e r i a l i e n ~ u n d ~ E n e r g i e, ~ A l b e r t-E i n s t e i n-S t r . ~ 15, ~ B e r l i n ~ 12489, ~}$ Germany

${ }^{\mathrm{b}}$ Department of Chemistry, Moscow State University, Moscow 119991, Russian Federation

${ }^{c}$ Institute of Microelectronics Technology and High-Purity Materials Russian Academy of Sciences, Chernogolovka 142432, Russian Federation
\end{abstract}

Corresponding authors:

* Dr. habil. Maria Brzhezinskaya

Helmholtz-Zentrum Berlin für Materialien und Energie,

Albert-Einstein-Str. 15, 12489 Berlin, Germany

E-mail: maria.brzhezinskaya@helmholtz-berlin.de

** Prof. Dr. Dmitry Roshchupkin

Institute of Microelectronics Technology and High-Purity Materials Russian Academy of Sciences

Academician Ossipyan Str. 6

Chernogolovka 142432, Russian Federation

E-mail: rochtch@iptm.ru

\begin{abstract}
In the present work, it is demonstrated for the first time that a simple, specially developed method for graphene oxide (GO) deposition on large areas opens the prospects of GO's wide application in planar-group technologies for creating different electronic devices including memristor devices for neuromorphic computing systems in the field of large data and artificial intelligence. MOS structures based on synthesized large-area GO films were formed, and their switching characteristics were studied. Current-voltage measurements performed on the MOS capacitors demonstrated forming-less, device's self-limited current behavior of GO bipolar resistive switching characteristics with the current density of up to $1 \mathrm{~A} / \mathrm{cm}^{2}$. Multiple sharp transitions from the high resistance state to low resistance state in the pristine GO film under the DC voltage sweep may indicate formation of multiple conductive filaments that provide
\end{abstract}


stable conductive paths between GO layers. It was found out that in our devices at least two resistive switching mechanisms may occur simultaneously, namely, filament formation and charge trapping/de-trapping, which is great advantages of GO over other materials. The observed effects are interpreted using a model explaining resistive switching in GO associated with the drift of functional groups and its impact on the resulting different $s p^{3}$ and $s p^{2}$ domains.

Keywords: graphene oxide, resistive switching, memristor, non-volatile memory, core-level spectroscopy

\section{Declarations of interest: none.}

\section{Introduction}

Increase in energy consumption and decrease in the device size in modern microelectronics requires development of a new concept for the basic hardware components [1]. Non-volatile resistive memory devices allow creation of efficient systems for analog and neuromorphic computation in the field of large data and artificial intelligence. Reduction of material dimensions to the level of the atomic layer leads to unique physical and chemical properties exemplified by the properties of two-dimensional $(2 D)$ materials, including graphene and graphene oxide [2].

Among various carbon structures (carbon nanotubes, fullerenes, graphite, diamond, etc.), graphene is a monatomic two-dimensional $s p^{2}$-hybridized carbon layer with unique physical properties [3, 4]. Graphene attracted a great attention immediately after its discovery in 2004 [5]. This material is characterized by a high charge carrier mobility that allows it to be used as a channel in field-effect transistors [6]. It has been found out that graphene has no gap within the energy band which makes it unsuitable for digital electronics. However, the possibility of obtaining various modifications of graphene opens prospects for its application in different spheres of electronics [7]. Field-effect transistors [8-9], transparent conducting electrodes for OLED, photovoltaic and optoelectronic devices [10-11], Hall sensors [12] have already been created based on graphene.

Preparation of different graphene derivatives (e.g. graphene oxide (GO), graphene nitride (GN), graphene fluoride (GF)) considerably expanded the sphere of the $2 D$ crystals application in electronics. Graphene oxide has been used to produce transistors [13] and gauges [14]. The unique electronic and optical properties of graphene oxide demonstrate a huge potential for designing ultrahigh density memristive elements to be used as interfaces with electronic 
artificial neural networks. The different degree of reduction/oxidation of graphene oxide/graphene allows monitoring multiple memristive states in a wide spectral range by using electric and optic excitation [15]. Non-volatile resistive memory (NVM) devices allow creation of efficient systems for analog and neuromorphic computation in the field of large data and artificial intelligence [16]. Graphene oxide (GO)-based NVM devices were used to evaluate spike-time-dependent plasticity (STDP) to construct an artificial synapse [17]. However, a limited number of investigations on the GO memristive properties were performed. Despite the rapid increase of number of publications on GO-based memristors, due to the complex structure of a thick GO film, a highly functionalized form of graphene that is usually stacked in platelets [18], the operative switching mechanism of GO has not yet been clearly understood also [19, 20]. To explain the effect of the GO resistive switching three major models have been discussed [21]: metallic-based filamentary conduction [22], contact-resistance modification caused by ion drift [23] and oxidation-reduction mechanisms in the bulk GO [24].

In this work, we demonstrated for the first time that a simple, specially developed method for graphene oxide deposition on large areas opens the prospect of wide application of graphene oxide in planar-group technologies for creating different electronic devices including memristor devices for neuromorphic computing systems. For this purpose, the electrical properties of large-area GO films with flakes a few microns in size were studied. MOS structures based on synthesized large-area GO films were formed and switching characteristics of GO films were investigated. The current-voltage measurements performed on the MOS capacitors demonstrated forming-less bipolar resistive switching characteristics with the current density up to $1 \mathrm{~A} / \mathrm{cm}^{2}$. It was found that two resistive switching mechanisms may operate simultaneously in our devices, which is great advantages of GO over other materials. In parallel with this, the synthesized GO/Si samples were comprehensively investigated by scanning electron microscopy and infrared (IR), Raman, high-resolution synchrotron-based core-level spectroscopies to define the features of their atomic and electronic structures and to give explanation for observed new effects.

\section{Material and methods}

Graphene oxide was synthesized by oxidation of graphite using the modified Hummers method $[25,26]$. This method was characterized by a long oxidation time and highly effective procedure for purifying reaction products was used. Native graphite (Aldrich graphite, 99.995 wt $\%$, high density) was added to a mixture of concentrated sulfuric acid $\left(\mathrm{H}_{2} \mathrm{SO}_{4}\right)$ and phosphoric acid $\left(\mathrm{H}_{3} \mathrm{PO}_{4}\right)$ and dispersed. During the process, potassium permanganate $\left(\mathrm{KMnO}_{4}\right)$ 
was gradually added to the mixture as an oxidant. The mixture was maintained for 36 hours at room temperature under continuous stirring. Then, aqueous solution of hydrogen peroxide $\left(\mathrm{H}_{2} \mathrm{O}_{2}\right)$ was added and the mixture was stirred for 30 minutes. The resulting mixture was then added to an aqueous solution of $\mathrm{H}_{2} \mathrm{SO}_{4}$ with stirring for 20 minutes, followed by stirring for 1 hour. The mixture was sifted through two standard metal test sieves with cell diameters of 320 $\mu \mathrm{m}$ and $30 \mu \mathrm{m}$. Unexfoliated particles were removed by centrifuging at $9000 \mathrm{rpm}$ for 20 minutes. The remaining solid material was then washed successively with hydrochloric acid, distilled water, and ethanol. The resulting GO suspension was dried in vacuum at $60{ }^{\circ} \mathrm{C}$.

The graphene oxide films $(\mathrm{GO} / \mathrm{Si})$ were formed on the $\mathrm{Si}$ substrates by spin-coating the GO based sol of $5 \mathrm{mg} / \mathrm{ml}$ at the rotation speed of $3000 \mathrm{rpm}$.

The atomic and electronic structures of synthesized GO/Si samples were characterized by scanning electron microscopy, as well as by infrared, Raman, X-ray absorption and photoelectron spectroscopies.

The scanning electron microscopy (SEM) images were recorded using Carl Zeiss Supra 40 (Germany).

The transmission-mode IR spectra were detected in the range of $400-4000 \mathrm{~cm}^{-1}$ using a Frontier Perkin Elmer spectrometer.

The Raman spectra of GO/Si and graphene samples were recorded using the SENTERRA Bruker Raman microscope at the laser wavelength of $532 \mathrm{~nm}$.

The high-resolution X-ray absorption and photoelectron spectra of $\mathrm{GO} / \mathrm{Si}$ and graphene samples were measured using the facilities of the Russian-German beamline (RGBL) at the BESSY II synchrotron radiation source of Helmholtz-Zentrum Berlin (HZB) [27]. The measurements were carried out under ultra-high vacuum conditions ( $\mathrm{P} \sim 2 \times 10^{-10}$ Torr). The NEXAFS spectra were obtained by recording the total electron yield (TEY) in the mode of measuring the sample drain current. The monochromator energy resolution near the $\mathrm{C} 1 \mathrm{~s}$ absorption edge ( $285 \mathrm{eV})$ was $\sim 70 \mathrm{meV}[28]$.

The X-ray photoemission spectra were acquired using a Phoibos 150 electron energy analyzer (Specs). The $\mathrm{C} 1 s$ and $\mathrm{O} 1 \mathrm{~s}$ spectra were acquired at the photon energy $(h v)$ of $850 \mathrm{eV}$ and analyzer pass energies $(P E)$ of $20 \mathrm{eV}$ with the overall (monochromator and analyzer) energy resolution of $\sim 300 \mathrm{meV}$. The $\mathrm{C} 1 \mathrm{~s}, \mathrm{Au} 4 f$ and valence band spectra of the reference samples (HOPG and $\mathrm{Au}$ ) were measured to calibrate the analyzer work function. The detection angle was close to normal emission. For the data analysis, the spectra were fitted by the Gaussian/Lorentzian convolution functions with simultaneous optimization of the background parameters using the UNIFIT software [29]. 
The GO film switching characteristics were studied by the DC current-voltage measurements using Keithley 2450 SourceMeter on the MOS structures (Fig. 1(a)) made on five phosphorous doped Si wafer pieces with the GO film thickness in the range from 15 to $40 \mathrm{~nm}$. The top electrodes with the size of $1 \mathrm{~mm}^{2}$ were made by sputtering $\mathrm{Al}$ and the bottom electrode was formed by scratching InGa paste.

\section{Results and discussion}

To comprehensively characterize the synthesized GO/Si samples and to verify their high quality, scanning electron microscopy and infrared (IR), Raman, X-ray absorption and photoelectron spectroscopies were used.

First of all, scanning electron microscopy was used to verify the quality of the samples after synthesis. The SEM definitely demonstrates that a uniform large-area GO film was formed on the $\mathrm{SiO}_{2} / \mathrm{Si}$ surface with flake sizes of 2-6 $\mu \mathrm{m}$ (Fig. 1(b)). The thickness of the GO film was controlled by an atomic force microscopy on a step formed on the witness sample during the formation of GO film under the same conditions as the sample for the $I-V$ measurements was fabricated.

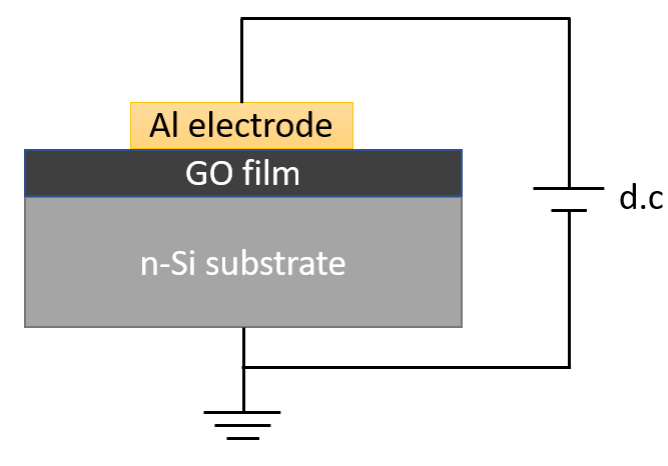

(a)

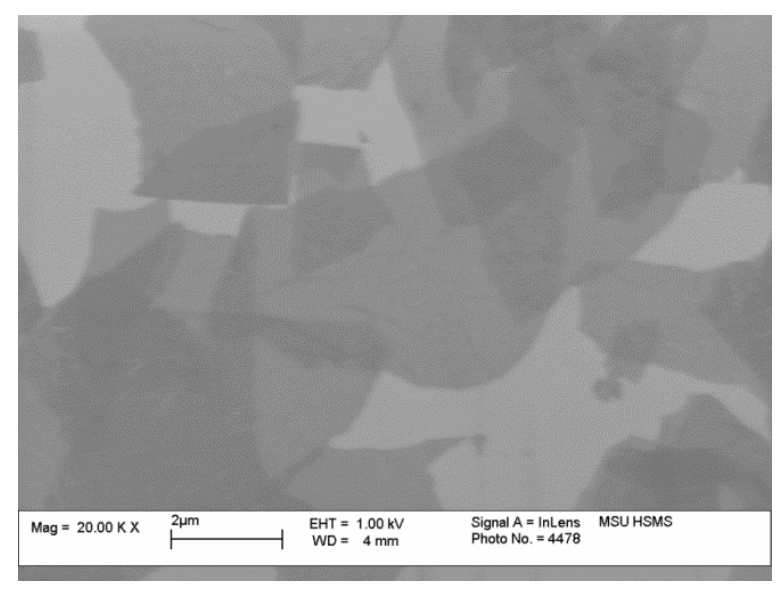

(b)

Fig. 1. Schematic of MOS capacitor (a) and SEM image of GO sheets (b).

Then, the efficiency of GO oxidation was controlled using IR spectroscopy. Figure 2 shows the typical IR spectrum of the GO film. A number of vibrations from oxygen groups are clearly seen $[30,31]$ : COOH/O-H stretching $\left(3600 \mathrm{~cm}^{-1}\right), \mathrm{H}-\mathrm{O}-\mathrm{H}$ stretching $\left(3400 \mathrm{~cm}^{-1}\right), \mathrm{C}=\mathrm{O}$ 
stretching $\left(\sim 1720 \mathrm{~cm}^{-1}\right), \mathrm{H}-\mathrm{O}-\mathrm{H}$ deformation $\left(1620 \mathrm{~cm}^{-1}\right), \mathrm{C}=\mathrm{C}$ stretching $\left(1580 \mathrm{~cm}^{-1}\right)$ and CO stretching (a number of peaks in the range of 1460-1000 $\mathrm{cm}^{-1}$ ).

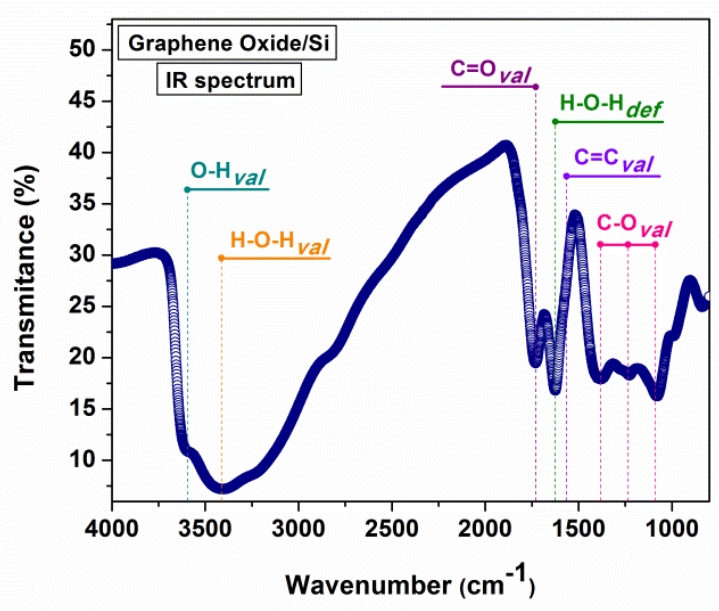

Fig. 2. IR spectrum of GO/Si sample.

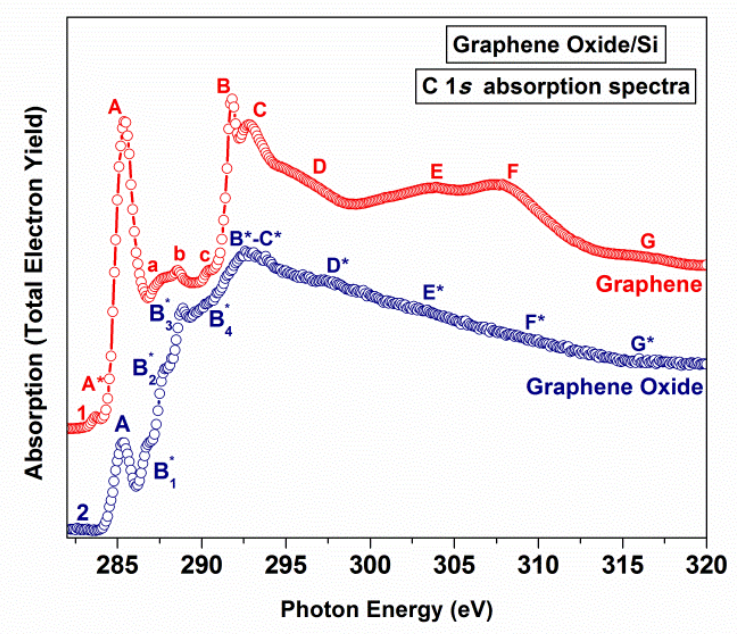

Fig. 3. The $\mathrm{C} K$ spectra of $\mathrm{GO} / \mathrm{Si}$ and graphene/LGT.

After that the electronic structure of the above-mentioned GO samples was investigated using high-resolution X-ray absorption and photoelectron spectroscopies. The GO core-level spectra are presented in Figs. 3 and 4. Figure 3 compares the $C K$ spectrum of graphene oxide with the spectrum of the pristine graphene (graphene/La $\mathrm{La}_{3} \mathrm{Ga}_{5.5} \mathrm{Ta}_{0.5} \mathrm{O}_{14}$ ) [32]. The $\mathrm{C} 1 s$ spectra were registered at the angle of $54.7^{\circ}$ (magic angle) between the normal and polarization vector of plane-polarized synchrotron radiation in order to avoid the effect of the orientation dependence of the absorption spectra. The $\mathrm{C} 1 s$ spectrum of the initial graphene agrees with the data reported in other works [33-35]. According to these data, peaks $A$ and $B-C$ in the graphene spectrum are appeared due to the dipole-resolved transition of $\mathrm{C} 1 s$-electrons to the free states of $\pi$ - and $\sigma$-symmetry of the conduction band which are formed from the $\pi 2 p_{\mathrm{z}^{-}}$and $\sigma 2 p_{\mathrm{x}, \mathrm{y}}$-states of the carbon atom oriented vertically to and laterally in the plane of the graphene layer [32]. The low-energy step $A^{*}(h v=283.7 \mathrm{eV})$ is a distinguishing feature of the near-edge fine structure of the X-ray absorption spectra of single- and double-layer graphene and is a consequence of the formation of additional electronic states due to arising of a chemical bond between graphene and substrate atoms (in our case, $\mathrm{La}_{3} \mathrm{Ga}_{5.5} \mathrm{Ta}_{0.5} \mathrm{O}_{14}$ ), which results from hybridization of the valence electronic states of the substrate atoms and $2 p_{z} \pi$ electrons of graphene carbon atoms. The absorption bands $D-F$ represent the electron transitions to the free $\sigma$ states in the conduction band and are connected with the interaction of carbon hexagons in the graphene layer. The 
features of $a, b, c$ are due to the transitions of $\mathrm{C} 1 s$ electrons to the free $2 p$ states of carbon atoms in the graphene mesh areas oxidized during their synthesis.

The graphene oxide $\mathrm{C} 1 s$ absorption spectrum (Fig. 3) differs from the spectrum of the pristine graphene, which agrees with the data reported in other publications [36-42]. Several new spectral features appeared in the GO spectrum. This, first of all, concerns to peaks $B_{1} *-B_{3} *$ ( $h v=286.9 \mathrm{eV}, 287.9 \mathrm{eV}, 289 \mathrm{eV}$ ) appearing between the $\pi$ - and $\sigma$-resonances, and to feature $B_{4} *$ at the left side of the $\sigma$ resonance $B(h v=290.3 \mathrm{eV})$. Moreover, the $\pi$ - and $\sigma$-resonances $A$ and $B$ become weaker. On the other hand, the $\mathrm{C} 1 s$-spectrum of GO does not clearly exhibit the $E-G$ peaks. Oxidation leads to their broadening and decreasing of their intensity, as a result of which peaks $B$ and $C$ merge into one $B^{*}-C^{*}$ band similarly to the effect observed in the absorption spectra of fluorinated (F-SWNTs) and hydrogenated (H-SWNTs) single-wall carbon nanotubes $[43,44]$.

Table 1. Interpretation of spectral features in the $\mathrm{C} K$ spectrum of GO.

\begin{tabular}{cccc}
\hline Peak & Position, eV & Assignment & References \\
\hline$B_{1} *$ & 286.9 & $\begin{array}{c}\text { Hydroxyl } \\
\pi^{*} \mathrm{C}-\mathrm{OH}\end{array}$ & $\begin{array}{c}{[33],[35],[36],} \\
{[37]}\end{array}$ \\
\hline$B_{2} *$ & 287.9 & $\begin{array}{c}\sigma^{*} \text { Epoxy } \\
(\mathrm{C}-\mathrm{O})\end{array}$ & {$[35],[36]$} \\
\hline$B_{3}^{*}$ & 289.0 & $\begin{array}{c}\text { Carboxyl } \\
\pi^{*} \mathrm{C}(=\mathrm{O}) \mathrm{OH}\end{array}$ & $\begin{array}{c}\text { [35], [36], [37], } \\
{[38],[39]}\end{array}$ \\
\hline$B_{4} *$ & 290.3 & $\begin{array}{c}\text { Carbonyl } \\
\pi^{*} \mathrm{C}=\mathrm{O}\end{array}$ & {$[33],[35]$} \\
\hline
\end{tabular}

The new details in the GO C1s absorption spectrum undoubtedly arise due to the electron transitions into new states in the conduction band where the states are formed during graphene oxidation both on its edges and directly in the layer plane. The states resulting from the chemical bonding between carbon and oxygen atoms cause a rearrangement of the GO structure, which, however, does not induce changes in the carbon atoms hybridization. Table 1 gives data on the identification of new spectral features appearing in the spectrum of graphene as a result of its oxidation. By analogy with other authors, features $B_{1} *(h v=286.9 \mathrm{eV}), B_{3}^{*}(h v=289.0 \mathrm{eV})$, and $B_{4}^{*}(h v=290.3 \mathrm{eV})$ can be identified as due to transitions of core $\mathrm{C} 1 s$ electrons to the $\pi^{*}$ symmetry states mainly localized to $\mathrm{C}-\mathrm{O}$ bonds of hydroxyl groups $(\mathrm{C}-\mathrm{OH})[36,38,39,41]$, $\mathrm{C}=\mathrm{O}$ bonds of carboxylic acid $(-\mathrm{COOH})$ [38-42], and carbonyl groups $(\mathrm{C}=\mathrm{O})[36,38]$, respectively. This identification of the new states agrees with the Lerf-Klinowski model of graphene oxide according to which epoxide and hydroxyl groups are positioned over the basic 
graphene plane whereas ketones and carboxyl groups arise on the edges of the graphene layer $[45,46]$. All these properties point to a high quality of our sample and allow the sample to be characterized as a graphene oxide.

The results of our studies of the GO sample by X-ray absorption spectroscopy completely coincide with the data obtained by X-ray photoelectron spectroscopy. Figure 4 shows C $1 s$ (Fig. 4(a)) and O1s (Fig. 4(b)) photoelectron spectra for GO and C $1 s$ photoelectron spectrum of graphene (Fig. 4(a), inset), which were measured using photons with the excitation energy of $850 \mathrm{eV}$ that provided both optimal energy resolution and bulk sensitivity of $\sim 1.1 \mathrm{~nm}$. The $\mathrm{C} 1 \mathrm{~s}$ photoelectron spectrum of graphene (Fig. 4a, inset) has a well-recognized main peak located at the binding energy $(B E)$ of $284.8 \mathrm{eV}$. The low-intensity component at $B E=286 \mathrm{eV}\left(\mathrm{C}-\mathrm{O}_{\mathrm{x}}\right)$ may be related to oxidation. The main peak in the graphene $\mathrm{C} 1 s$ photoelectron spectrum is accompanied also by a satellite whose position with respect to the $\mathrm{C} 1 s$ peak is $6.1 \mathrm{eV}(B E=290.9$ $\mathrm{eV}$ ). The satellite is associated with discrete energy losses of emitted $\mathrm{C} 1 s$ photoelectrons for exciting valence electrons in the graphene layer [28].
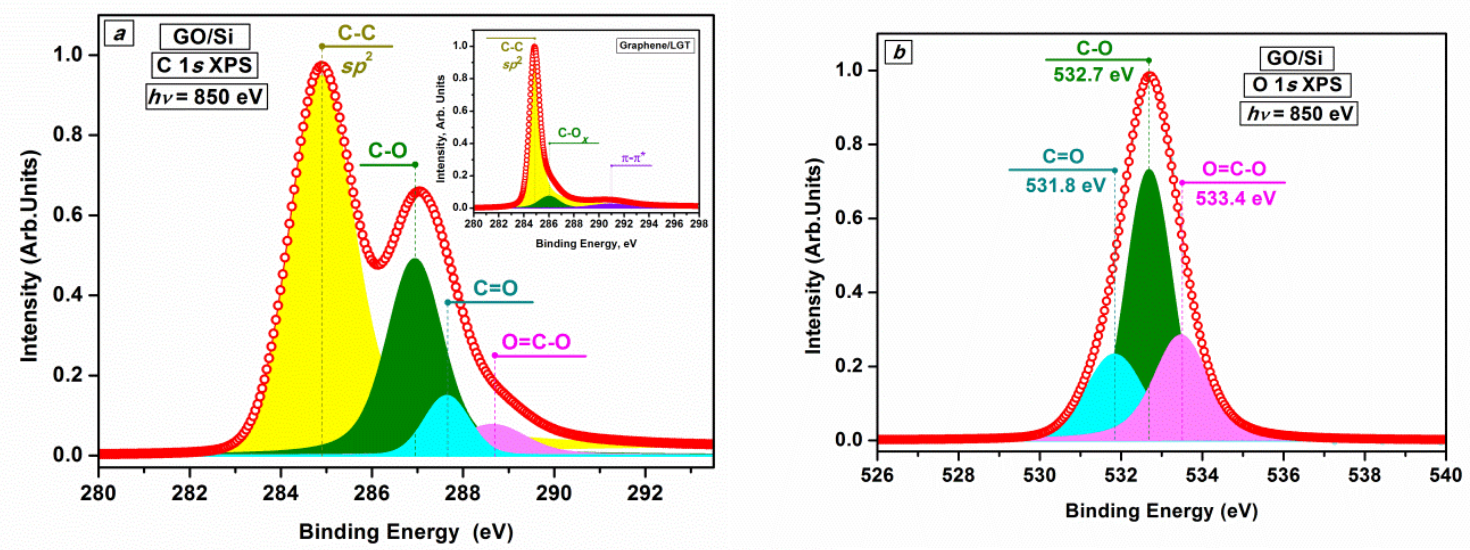

Fig. 4. $\mathrm{C} 1 s$ photoelectron spectra of the GO and graphene (inset) (a), and $\mathrm{O} 1 s$ photoelectron spectra of the GO (b), measured at $h v=850 \mathrm{eV}$.

Unlike the spectrum of graphene, the $\mathrm{C} 1 s$ photoelectron spectrum of graphene oxide (Fig. 4(a)) has a multicomponent structure and may be well fitted by four peaks. The main peak (in yellow color), which, like that in graphene, is located at $B E=284.8 \mathrm{eV}$ and has an asymmetric shape on the side of high binding energies. Also, as in the case of graphene, it is described using the Doniach-Sunjic function with an asymmetry coefficient $\alpha=0.1$. Therefore, it can be uniquely identified with a graphene $\mathrm{C}=\mathrm{C}$ phase with $s p^{2}$ hybridization of the valence states of the carbon atom. The other three peaks have a symmetrical shape and are located on the high-energy side 
of the main peak. The relative intensities of the fitting peaks estimated by comparing the areas under the peaks are 62.5: 26.8: 5.8: 4.9, respectively. Arising of these three peaks in the GO $\mathrm{C} 1 s$ spectrum, as compared with the graphene spectrum, is obviously associated with the graphene oxidation and could therefore be considered as a consequence of the chemical bonding between carbon and oxygen atoms. This interaction leads to the formation of oxygen-containing functional groups and is accompanied by the transfer of a charge from carbon to oxygen atoms due to the greater electronegativity of the latter. These three peaks have binding energies of $286.9 \mathrm{eV}$ (shaded in green), $287.6 \mathrm{eV}$ (in blue), and $288.6 \mathrm{eV}$ (in pink) corresponding to chemical shifts of $2.1 \mathrm{eV}, 2.8 \mathrm{eV}$ and $3.8 \mathrm{eV}$. Therefore, they can be attributed to C-O (epoxy and hydroxyl), $\mathrm{C}=\mathrm{O}$ (carbonyl) and $\mathrm{O}=\mathrm{CO}$ - (carboxyl) types of bonds, respectively, which we have already identified above in the analysis of the $\mathrm{C} 1 s$ absorption spectrum of GO (Fig. 3), which also agrees with the data of other authors [47-52].

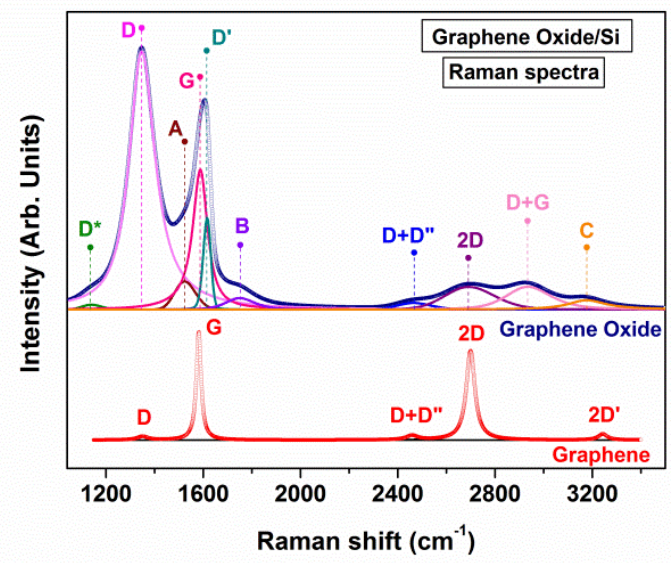

Fig. 5. Raman spectra of graphene/LGT and GO/Si, measured at $\lambda=532 \mathrm{~nm}$.

Figure $4 \mathrm{~b}$ shows the $\mathrm{O} 1 s$ photoelectron spectrum of GO. Unlike the $\mathrm{C} 1 s$ photoelectron spectrum of GO, this spectrum exhibits only one broad peak which can also be fitted by three components with binding energies of $531.8 \mathrm{eV}, 532.7 \mathrm{eV}$, and $533.4 \mathrm{eV}$. Differences between them correspond to the chemical shifts of $0.9 \mathrm{eV}$ and $1.6 \mathrm{eV}$. Therefore, they can be attributed to $\mathrm{C}=\mathrm{O}$ (carbonyl), $\mathrm{C}-\mathrm{O}$ (epoxy and hydroxyl) and $\mathrm{O}=\mathrm{C}-\mathrm{O}-$ (carboxyl) types of bonds, respectively [47-52], as was earlier done after the analysis of the GO C1s photoelectron spectra (Fig. 4(a)). Noteworthy is that the chemical shift between the similar peaks in the O1s photoelectron spectrum of GO is smaller than that in the $\mathrm{C} 1 s$ photoelectron spectrum. It should be noted that $1 s$ photoelectron spectra of oxygen atoms are less informative than spectra of carbon. The effective charge of the oxygen atom in GO, like in most other compounds, is negative and changes with small changes in the atom chemical state. In our case, because of the 
oxygen atom participation in chemical binding with carbon atoms, slight changes in the density of its valence electrons is accompanied by a slight change in the screening of the skeleton $\mathrm{O} 1 \mathrm{~s}$ electrons by valence electrons, which results in small values of chemical shifts in the binding energies of $1 s$ electrons of oxygen atoms.

Figure 5 presents the Raman spectra recorded from GO and graphene samples. The experimental GO spectra were fitted with a combination of peaks of a flexible pseudo-Voigt shape [53]. Since the line broadening in disordered systems is not necessarily homogeneous, the peaks may be slightly asymmetrical. For the first-order spectral range, the fitting procedure was done according to the approach reported in [54] (i.e. 5 bands including $D^{*}, D, A, G$ and $D$ ') with one extra band at $\sim 1750 \mathrm{~cm}^{-1}(B)$ often found in graphene oxide as shown in [55]. The second-order spectral range was fitted with four peaks ( $D+D$ ', $2 D, D+G, C[56])$. The fitted peak characteristics of the GO film and graphene are collected in the tables 2 and 3, respectively. The $D$ peak is the LO mode close to the $K$ point in the Brillouin zone. The $G$ peak is the LO mode at the $\Gamma$ point. A significant broadening of the $D$ and $G$ peaks in graphene oxide, compared with graphene, is associated with an increase in the defect concentration caused by the oxidation of graphene. $D^{\prime}, 2 D$ and $D+G$ bands are also defect-related modes.

Table 2. Raman characteristics of GO.

\begin{tabular}{ccccc}
\hline Peak & $\Omega, \mathrm{cm}^{-1}$ & FWHM $\mathrm{cm}^{-1}$ & Intensity & Area \\
\hline$D^{*}$ & 1140 & 94 & 146 & 14674.3 \\
\hline$D$ & 1347.1 & 128 & 7801 & 1538970 \\
\hline$A$ & 1523.4 & 100 & 849.5 & 90829.3 \\
\hline$G$ & 1587.8 & 73 & 4227 & 482309.4 \\
\hline$D^{\prime}$ & 1616 & 37 & 2766 & 116177.2 \\
\hline$B$ & 1749.8 & 140 & 347.7 & 64535.3 \\
\hline$D+D^{\prime \prime}$ & 2465.3 & 163 & 200 & 37254.1 \\
\hline $2 D$ & 2692.4 & 240 & 686 & 188234.8 \\
\hline$D+G$ & 2935 & 216 & 690.7 & 197303.7 \\
\hline$C$ & 3177.1 & 189 & 282 & 84297.8 \\
\hline
\end{tabular}

In work [54], the $A$ peak was attributed to amorphous carbon. In previous works, peak $D^{*}$ was associated with the graphene mesh disordering due to $s p^{2}-s p^{3}$ bonds at the edges $[57,58]$. Peak $B$ is attributed to Stone Wales defects [59]. The $C$ band is usually labeled $2 D^{\prime}$. In graphene, 
the $2 D^{\prime}$ peak frequency is equal to twice the $D^{\prime}$ peak frequency. However, in graphene oxide, the frequency of peak $C$ is substantially less than twice the $D^{\prime}$ frequency. Work [55] proposed that this $C$ peak corresponds to the $\mathrm{C}-\mathrm{H}$ stretching mode of aromatic $\mathrm{C}$.

In work [54], it was demonstrated that the peak positions of the $A$ and $D^{*}$ bands depend on the oxygen content in graphene oxides. Using these results, we estimated the oxygen content in our GO films. We obtained a value equal to $30-35 \%$. This value is in good agreement with the oxygen content estimate of $37.5 \%$ derived from XPS data. In work [56], it was shown that the ratio between the $D$ and $G$ peak intensities and frequencies of the $2 D$ and $D+G$ bands linearly correlate with the $\mathrm{Csp} p^{2}$ content. In our GO films, these correlations lead to the Csp $p^{2}$ values of $56-65 \%$, which is also in good agreement with XPS data.

Table 3. Raman characteristics of graphene.

\begin{tabular}{ccccc}
\hline Peak & $\omega, \mathrm{cm}^{-1}$ & FWHM, $\mathrm{cm}^{-1}$ & Intensity & Area \\
\hline D & 1350.9 & 50.9 & 108.2 & 8646 \\
\hline G & 1582 & 23.6 & 3260.5 & 120860 \\
\hline D+D', & 2459.2 & 55.7 & 129 & 11295.5 \\
\hline 2D & 2699.9 & 42.5 & 2693.4 & 179895.6 \\
\hline 2D' & 3243.7 & 43.8 & 180 & 12403 \\
\hline
\end{tabular}

To reveal the defect type dominating in our GO films, we calculated the ratio between areas under the $D$ and $D$ ' bands $\left(A_{\mathrm{D}} / A_{\mathrm{D}}\right)$. It appeared to be 0.075 . This means that defects with $s p^{3}$ hybridization predominate in the films [60]. Thus, our results agree with the graphene oxide cluster model, according to which GO consists of $s p^{2}$ graphene domains in an oxygen-enriched $s p^{3}$ matrix [55].

In this work, the current-voltage measurements on the large flake size graphene oxide and $n$-type Si based MOS capacitors have been performed for the first time. The measurements demonstrated forming-less, device self-limited current behavior of the GO bipolar resistive switching characteristics (Fig. 6). Under a positive DC voltage sweep the virgin GO film shows low current density up to $2 \mathrm{~V}$ reaching the value of $10^{-6} \mathrm{~A} / \mathrm{cm}^{2}$ (Fig. 6(a)) and several sharp transitions from the high resistance state (HRS) to the low resistance state (LRS) can be seen in the 2 to $4 \mathrm{~V}$ voltage range. The total internal current gain is up to 5 orders of magnitude without a catastrophic breakdown of the GO film. Under the subsequent negative voltage sweep the current is notably lower than expected for the low resistance state (Fig. 6(a), curves 2 and 4). As the negative bias increases above $\sim 7 \mathrm{~V}$, several sharp transitions to the lower resistance state 
occur. This would appear to be due to volatility of the GO film. However, at the negative bias the GO film is in series with a space charge region of the n-type silicon substrate, hence the current at a given voltage is significantly reduced. Thus, the GO film demonstrated resistive switching from HRS to LRS under positive voltage, while under negative bias the transition to even lower resistance state occurs. When the voltage sweep was repeated only towards one polarity, HRS to LRS transition did not occur, thus indicating a non-volatile behavior. Figure 6(b) shows the switching characteristics plotted in a linear scale. It is worth to note that the LRS current is strongly non-linearly dependent on the applied voltage at both polarities.

Similar results demonstrating resistive switching from HRS (or some intermediate resistance state) to LRS under both voltage polarities were also observed when a virgin MOS capacitor was first swept with a negative voltage. As can be seen from Fig. 6(c), a sharp HRS to LRS transitions occur at voltages of about negative $2 \mathrm{~V}$ and negative $4 \mathrm{~V}$. As the negative voltage further increases, the current keeps gradually rising until the low resistance state is reached at about negative $9 \mathrm{~V}$. The subsequent positive voltage sweeps results in a gradual transition from the intermediate resistance state to the LRS.
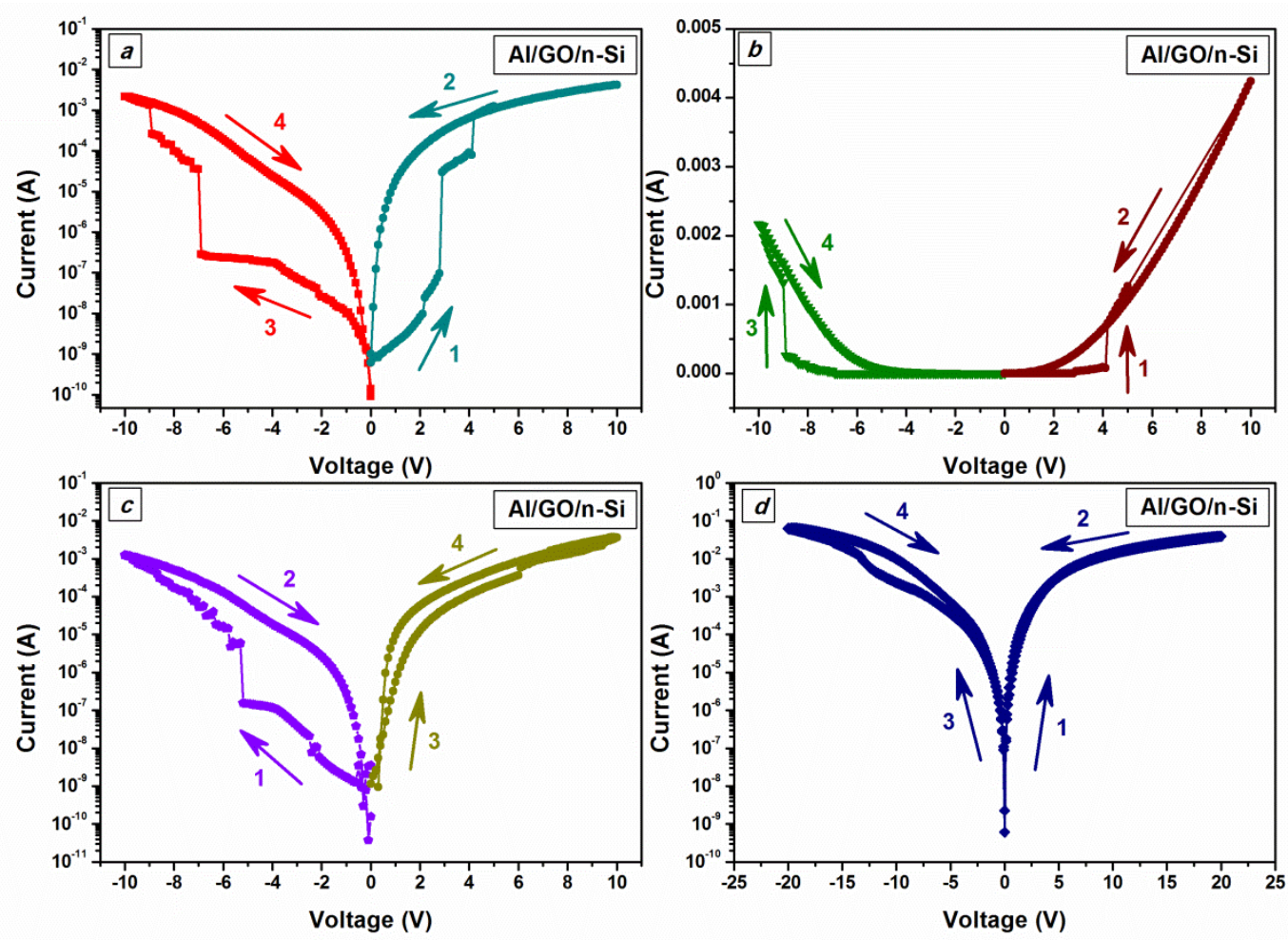

Fig. 6. I-V characteristics of GO based MOS structures with a $15 \mathrm{~nm}$ thick GO film under DC voltage sweep: (a) and (b) first DC cycle started with a positive voltage (c) first DC cycle started with a negative voltage, (d) I-V curve after multiple DC cycles. Numbered arrows show voltage sweep direction. 
The current compliant-less switching characteristics showed in Figures 6(a) and 6(c) are rather well repeatable during at least 20 DC cycles without a notable variation of the LRS values. However, some virgin MOS devices with a substantially lower initial resistance revealed quite different behavior, namely, after about 10 DC cycles the resistive switching disappeared during the positive voltage sweep, whereas the negative voltage still forced the HRS to LRS transition (Fig. 6(d)). When the voltage sweep was repeated only towards the negative values, LRS to HRS transition did not occur thus indicating a non-volatile behavior. This interesting feature can be related either to a GO thickness or structure variation within a rather large $\left(1 \mathrm{~mm}^{2}\right)$ device size. The switching characteristics presented in Fig. 6 were obtained on the MOS structures with a $15 \mathrm{~nm}$ thick GO film. Similar results were obtained on the MOS structures with a $20 \mathrm{~nm}$ thick GO film, while the GO films thicker than $20 \mathrm{~nm}$ demonstrated less stable switching behavior and required application of a much higher, up to $15-20 \mathrm{~V}$, voltage.

The above features of GO resistive switching contribute to a better understanding of an operative switching mechanism. The HRS to LRS transition in the pristine GO film under the positive voltage sweep may indicate the formation of multiple conductive filaments that provide stable conductive paths between GO layers. Such filaments consisting of the $s p^{2}$ domains in $s p^{3}$ domain can be formed under electric field [61]. In our MOS devices, the filaments appear to be not broken off when the negative bias is applied. Moreover, the Fig. 6(a) data may indicate that even more conductive filaments are being formed during the negative voltage sweeping. At the same time, the data in Fig. 6(d) demonstrate that stable non-volatile switching is achievable when the voltage is swept to both the negative and positive values. This can be explained by capturing and emitting the electrons by deep level traps existing in the GO film. The data in Figures 6(a)-6(c) showing two HRS to LRS set transitions may indicate either injection of holes from the substrate and their trapping within the GO film or formation of a back-to-back structure between two parallel flakes. The charge trapping effect was observed via the time and temperature dependent capacitance-voltage measurements (not shown in this paper). Thus, we have found out that in our devices at least two resistive switching mechanisms, namely, filament formation and the charge trapping/de-trapping, may occur simultaneously in the GO film. According to the metallic filamentary conduction model [22], when a positive voltage is applied to the top Al electrode, due to its electrochemical oxidation, the resulting cations migrate through the insulating layer towards the cathode thus forming a filament. Under the reversed bias polarity, a reverse diffusion is supposed to take place resulting in the rupture of the metallic filament. Our experimental results showing HRS to LRS transitions under both voltage 
polarities obviously do not support this model. The non-linear current voltage dependence in the LRS in our experiments may indicate a lack of a conducting filament in the bulk GO as suggested by the model of contact resistance modification caused by oxygen ion drift [23]. In this model, the resistive switching was attributed to the formation and rupture of local filaments in the thin insulating layer at the interface between the top Al electrode and the bulk GO film. This region is expected to be rich in $s p^{3}$ insulating domains constituting an insulating barrier that gives rise to the HRS. Under reverse bias polarity, local conductive filaments are grown by field-induced oxygen diffusion into the GO; thus, the oxygen returns back to the bulk GO decreasing the insulating barrier and giving rise to the LRS. However, this model assumes the voltage polarity dependent HRS to LRS transition that was not observed on our case. The third model explaining resistive switching in GO belongs to the drift of functional groups and its impact on the resulting different $s p^{3}$ and $s p^{2}$ domains on the bulk substrates [24]. This is particularly relevant to describe planar structures. In this model, resistive switching is connected to a reversible filamentary phenomenon [62] in which the conductive path or paths are formed by turning insulating $s p^{3}$ domains into conducting $s p^{2}$ ones due to the detachment of oxygen containing groups under the action of an electric field. It appears that our experimental results agree with this model as it does not require specific voltage polarity dependence.

Thus, we have found out that in our devices at least two resistive switching mechanisms, filament formation and the charge trapping/de-trapping, may occur simultaneously in the GO film. To obtain more information on the operative switching mechanisms in the future work we plan to investigate dependence of switching characteristics on the GO film thickness, its structure, and the top electrode size. The charge trapping effect that we plan to study in detail via the time, voltage and temperature dependent measurements of the current-voltage, capacitance-voltage characteristics along with applying the method of thermally stimulated current to determine the energy levels in the GO film, will allow us to separate contribution of different switching operative mechanisms. To further deconvolute the contribution of various mechanisms in the resistive switching behavior, the possible effect of hole injection from the $n$-Si substrate will be eliminated by depositing the bottom electrode to the oxidized Si wafer. Although detection and visualization of a conductive filament(s) is a difficult task, we believe that applying the methods of conductive atomic force microscopy (AFM) and electron beam induced current (EBIC) along with transmission electron microscopy (TEM) on the structures with various GO thickness and top electrode size may help to verify formation of conductive filaments between the GO flakes. 


\section{Conclusions}

In this work, we demonstrated for the first time that a simple, specially developed method for graphene oxide deposition on large areas opens the prospect of wide application of GO in planar-group technologies for creating different electronic devices including memristor devices for neuromorphic computing systems in the field of large data and artificial intelligence. For this purpose, the graphene oxide was synthesized by graphite oxidation. The synthesized GO/Si samples were comprehensively investigated by scanning electron microscopy and infrared (IR), Raman, high-resolution synchrotron-based X-ray absorption (XAS) and photoelectron (XPS) spectroscopies.

SEM demonstrated that a uniform large-area GO film was formed with flake sizes of 2-6 $\mu \mathrm{m}$. The efficiency of GO oxidation was controlled using IR spectroscopy and core-level spectroscopies. The data from X-ray photoelectron spectroscopy showing that the C-O interaction leads to the formation of oxygen-containing functional groups with $\mathrm{C}=\mathrm{O}$ (carbonyl), $\mathrm{C}-\mathrm{O}$ (epoxy and hydroxyl) and $\mathrm{O}=\mathrm{C}-\mathrm{O}-$ (carboxyl) types of bonds and is accompanied by the charge transfer from carbon to oxygen atoms. Analysis of Raman, X-ray absorption and photoelectron spectra demonstrated that the GO film under investigation consists of $s p^{2}$ graphene domains in an oxygen-enriched $s p^{3}$ matrix. MOS structures based on synthesized large-area GO films were formed, and their switching characteristics were studied. The currentvoltage measurements performed on the MOS capacitors demonstrated forming-less, device's self-limited current behavior of GO bipolar resistive switching characteristics with the current density of up to $1 \mathrm{~A} / \mathrm{cm}^{2}$. Multiple sharp transitions from the high resistance state to low resistance state in the pristine GO film under the DC voltage sweep may indicate formation of multiple conductive filaments that provide stable conductive paths between GO layers. Thus, it was found out that in our devices at least two resistive switching mechanisms may occur simultaneously, namely, filament formation and charge trapping/de-trapping, which is great advantages of GO over other materials. The observed effects were interpreted using a model explaining resistive switching in GO associated with the drift of functional groups and its impact on the resulting different $s p^{3}$ and $s p^{2}$ domains.

\section{Acknowledgements}

The work was done according to the state task of Ministry of Science and Higher Education of the Russian Federation (Project No. 075-00920-20-00). The work of O.O.K. was supported by the Russian Foundation for Basic Research (Project RFBR No. 18-29-06036). The work of S.K. was supported by the Russian Foundation for Basic Research (Project RFBR No. 
19-29-03035). We thank Helmholtz-Zentrum Berlin für Materialien und Energie for the allocation of synchrotron radiation beamtime.

\section{References}

[1] J. Borghetti, G.S. Snider, P.J. Kuekes, J.J. Yang, D.R. Stewart, R.S. Williams, 'Memristive' switches enable 'stateful' logic operations via material implication, Nature 464 (2010) 873-876. https://doi.org/10.1038/nature08940

[2] G.N. Panin, O.O. Kapitanova, Photomemristive heterostructures based on two-dimensional crystals, Adv. Mater. Lett. 10 (2019) 470-475. DOI: 10.5185/amlett.2019.2212

[3] K.S. Novoselov, A.K. Geim, S.V. Morozov, D. Jiang, M.I. Katsnelson, I.V. Grigorieva, S.V. Dubonos, A.A. Firsov, Two-dimensional gas of massless Dirac fermions in graphene, Nature 438 (2005) 197-200. https://doi.org/10.1038/nature04233

[4] F. Schedin, A.K. Geim, S.V. Morozov, E.W. Hill, P. Blake, M.I. Katsnelson, K.S. Novoselov, Detection of individual gas molecules adsorbed on graphene, Nat. Mater. 6 (2007) 652-655. https://doi.org/10.1038/nmat1967

[5] K.S. Novoselov, A.K. Geim, S.V. Morozov, D. Jiang, Y. Zhang, S.V. Dubonos, I.V. Grigorieva A.A. Firsov, Electric field effect in atomically thin carbon films, Science 306 (2004) 666-669. DOI: 10.1126/science. 1102896

[6] J. Chen, M. Ishigami, C. Jang, D.R. Hines, M.S. Fuhrer, E.D. Williams, Printed graphene circuits, Adv. Mater. 19 (2007) 3623-3627. https://doi.org/10.1002/adma.200701059

[7] A.T. Dideikin, A.Y. Vul, Graphene oxide and derivatives: the place in graphene family, Front. Phys. 6 (2019) 149. https://doi.org/10.3389/fphy.2018.00149

[8] M. C. Lemme, T. J. Echtermeyer, M. Baus, H. Kurz, A graphene field-effect device, IEEE Electron Device Lett. 28 (2007) 282-284. DOI: 10.1109/LED.2007.891668

[9] J. Kedzierski, P.-L. Hsu, P. Healey, P.W. Wyatt, C.L. Keast, M. Sprinkle, C. Berger, W.A. de Heer, A. Walt, Epitaxial graphene transistors on SiC substrates, IEEE Trans. Electron Devices 55 (2008) 2078-2085. DOI: 10.1109/TED.2008.926593

[10] G. Eda, G. Fanchini, M. Chhowalla, Large-area ultrathin films of reduced graphene oxide as a transparent and flexible electronic material, Nat. Nanotechnol. 3 (2008) 270-274. https://doi.org/10.1038/nnano.2008.83

[11] X. Li, W. Chen, S. Zhang, Z. Wu, P. Wang, Z. Xu, H. Chen, W. Yin, H. Zhong, 18.5\% efficient graphene/GaAs van der Waals heterostructure solar cell, Nano Energy 16 (2015) 310319. https://doi.org/10.1016/j.nanoen.2015.07.003 
[12] J. Dauber, A. A Sagade, M. Oellers, K. Watanabe, T. Taniguchi,; D. Neumaier, C. Stampfer, Ultra-sensitive Hall sensors based on graphene encapsulated in hexagonal boron nitride, Appl. Phys. Lett. 106 (2015) 193501. https://doi.org/10.1063/1.4919897

[13] C.-Y. Su, Y. Xu, W. Zhang, J. Zhao, A. Liu, X. Tang, C-H. Tsai, Y. Huang, L-J. Li, Highly efficient restoration of graphitic structure in graphene oxide using alcohol vapors, ACS Nano 4 (2010) 5285-5292. https://doi.org/10.1021/nn101691m

[14] Q. He, S. Wu, Z. Yin, H. Zhang, Graphene-based electronic sensors, Chem. Sci. 3 (2012) 1764-1772. https://doi.org/10.1039/C2SC20205K

[15] O.O. Kapitanova, G.N. Panin, D.C. Hak, A.N. Baranov, W.K. Tae, Formation of selfassembled nanoscale graphene/graphene oxide photomemristive heterojunctions using photocatalytic oxidation, Nanotechnology 28 (2017) 204005. https://doi.org/10.1088/1361$6528 / \mathrm{aa} 655 \mathrm{c}$

[16] M. Qi, S. Cao, L. Yang, Q. You, L. Shi, Z. Wu, Uniform multilevel switching of graphene oxide-based RRAM achieved by embedding with gold nanoparticles for image pattern recognition, Appl. Phys. Lett. 116 (2020) 163503. https://doi.org/10.1063/5.0003696

[17] L.Y. Wang, Z.Y. Wang, W. Zhao, B. Hu, L.H. Xie, M.D. Yi, H.F. Ling, C.X. Zhang, Y. Chen, J.Y. Lin, J. Zhu, W. Huang, Controllable multiple depression in a graphene oxide artificial synapse, Adv. Electron. Mater. $3 \quad$ (2017) 1600244. https://doi.org/10.1002/aelm.201770001

[18] M. Coroş, F. Pogăcean, L. Măgeruşan, C. Socaci, S. Pruneanu, A brief overview on synthesis and applications of graphene and graphene-based nanomaterials, Front. Mater. Sci. 13 (2019) 23-32. https://doi.org/10.1007/s11706-019-0452-5

[19] C. Tan, Z. Liu, W. Huang, H. Zhang, Non-volatile resistive memory devices based on solution-processed ultrathin two-dimensional nanomaterials, Chem. Soc. Rev. 44 (2015) 26152628. https://doi.org/10.1039/C4CS00399C

[20] S. Porro, E. Accornero, C.F. Pirri, C. Riccardi, Memristive devices based on graphene oxide, Carbon 85 (2015) 383-396. https://doi.org/10.1016/j.carbon.2015.01.011

[21] F.J. Romero, A. Toral, A. Medina-Rull, C.L. Moraila-Martinez, D.P. Morales, A. Ohata, A. Godoy, F.G. Ruiz, N. Rodriguez, Resistive switching in graphene oxide, Front. Mater. 7 (2020) 17. https://doi.org/10.3389/fmats.2020.00017

[22] F. Zhuge, B. Hu, C. He, X. Zhou, Z. Liu, R.W. Li, Mechanism of nonvolatile resistive switching in graphene oxide thin films, Carbon 49 (2011) 3796-3802. https://doi.org/10.1016/j.carbon.2011.04.071 
[23] H.Y. Jeong, J.Y. Kim, J.W. Kim, J.O. Hwang, J.E. Kim, J.Y. Lee, T.H. Yoon, B.J. Cho, S.O. Kim, R.S. Ruoff, S.Y. Choi, Graphene oxide thin films for flexible nonvolatile memory applications, Nano Lett. 10 (2010) 4381-4386. https://doi.org/10.1021/nl101902k

[24] G. Khurana, P. Misra, R.S. Katiyar, Forming free resistive switching in graphene oxide thin film for thermally stable nonvolatile memory applications, J. Appl. Phys. 114 (2013) 124508. https://doi.org/10.1063/1.4823734

[25] D.C. Marcano, D.V. Kosynkin, J.M. Berlin, A. Sinitskii, Z. Sun, A. Slesarev, L.B. Alemany, W. Lu, J.M. Tour, Improve synthesis of graphene oxide, ACS Nano 4 (2010) 48064814. https://doi.org/10.1021/nn1006368

[26] G.N. Panin, O.O. Kapitanova, S.W. Lee, A.N. Baranov, T.W. Kang, Resistive switching in Al/graphene oxide/Al structure, Jpn. J. Appl. Phys. $50 \quad$ (2011) 070110. https://doi.org/10.1143/JJAP.50.070110

[27] S.L. Molodtsov, S.I. Fedoseenko, D.V. Vyalikh, I.E. Iossifov, R. Follath, S.A. Gorovikov, M.M. Brzhezinskaya, Yu.S. Dedkov, R. Puettner, J.-S. Schmidt, V.K. Adamchuk, W. Gudat, G. Kaindl, High-resolution Russian-German beamline at BESSY, App. Phys. A 94 (2009) 501505. https://doi.org/10.1007/s00339-008-4916-1

[28] M.V. Kharlamova, L.V. Yashina, A.A. Volykhov, J.J. Niu, V.S. Neudachina, M.M. Brzhezinskaya, T.S. Zyubina, A.I. Belogorokhov, A.A. Eliseev, Acceptor doping of singlewalled carbon nanotubes by encapsulation of zinc halogenides, Eur. Phys. J. B 85 (2012) 34. https://doi.org/10.1140/epjb/e2011-20457-6

[29] A.A. Eliseev, L.V. Yashina, N.I. Verbitsky, M.M. Brzhezinskaya, M.V. Kharlamova, M.V. Chernysheva, A.V. Lukashin, N.A. Kiselev, A.S. Kumskov, B. Freitag, A.V. Generalov, A.S. Vinogradov, Y. Zubavichus, E. Kleimenov, M. Nachtegaal, Interaction between single walled carbon nanotube and 1D crystal in $\mathrm{CuX} @ \mathrm{SWNT}(\mathrm{X}=\mathrm{Cl}, \mathrm{Br}, \mathrm{I})$ nanocomposites, Carbon 50 (2012) 4021-4039. http://dx.doi.org/10.1016/j.carbon.2012.04.046

[30] T. Szabó, O. Berkesi, P. Forgó, K. Josepovits, Y. Sanakis, D. Petridis, I. Dékány, Evolution of surface functional groups in a series of progressively oxidized graphite oxides, Chem. Mater. 18 (2006) 2740-2749. https://doi.org/10.1021/cm060258+

[31] E. Pretsch, P. Bühlmann, M. Badertscher, Structure Determination of Organic Compounds. Tables of Spectral Data, Springer, Berlin, Heidelberg, Germany, 2009. DOI 10.1007/978-3662-04201-4

[32] M. Brzhezinskaya, A. Irzhak, D. Irzhak, T.W. Kang, O. Kononenko, V. Matveev, G. Panin, D. Roshchupkin, Direct growth of graphene film on piezoelectric $\mathrm{La}_{3} \mathrm{Ga}_{5.5} \mathrm{Ta}_{0.5} \mathrm{O}_{14}$ crystal, Phys. Status Solidi (RRL) 10 (2016) 639-644. https://doi.org/10.1002/pssr.201600122 
[33] D. Pacile, M. Papagno, A. Fraile Rodriguez, M. Grioni, L. Papagno, C. O. Girit, J. C. Meyer, G. E. Begtrup, A. Zettl, Near-edge X-ray absorption fine-structure investigation of graphene, $\quad$ Phys. $\quad$ Rev. $\quad$ Lett. $101 \quad$ (2008) 066806. https://doi.org/10.1103/PhysRevLett.101.066806

[34] B.J. Schultz, C. Jaye, P.S. Lysaght, D.A. Fischer, D. Prendergast, S. Banerjee, On chemical bonding and electronic structure of graphene-metal contacts, Chem. Sci. 4 (2013) 494-502. https://doi.org/10.1039/C2SC21018E

[35] E. Voloshina, R. Ovcharenko, A. Shulakov, Yu. Dedkov, Theoretical description of X-ray absorption spectroscopy of the graphene-metal interfaces, J. Chem. Phys. 138 (2013) 154706. https://doi.org/10.1063/1.4801501

[36] V. Lee, R. V. Dennis, B. J. Schultz, C. Jaye, D. A. Fischer, S. Banerjee, Soft X-ray absorption spectroscopy studies of the electronic structure recovery of graphene oxide upon chemical defunctionalization, J. Phys. Chem. C $116 \quad$ (2012) 20591-20599. https://doi.org/10.1021/jp306497f

[37] V. Lee, R.V. Dennis, C. Jaye, X. Wang, D.A. Fischer, A.N. Cartwright, S. Banerjee, In situ near-edge X-ray absorption fine structure spectroscopy investigation of the thermal defunctionalization of graphene oxide, J. Vac. Sci. Technol. B 30 (2012) 061206. https://doi.org/10.1116/1.4766325

[38] B.J. Schultz, R.V. Dennis, J.P. Aldinger, C. Jaye, X. Wang, D.A. Fischer, A.N. Cartwright, S. Banerjee, X-ray absorption spectroscopy studies of electronic structure recovery and nitrogen local structure upon thermal reduction of graphene oxide in an ammonia environment, RSC Adv. 4 (2014) 634-644. https://doi.org/10.1039/C3RA45591B

[39] H.-K. Jeong, H.-J. Noh, J.-Y. Kim, M.H. Jin, C. Y. Park, Y.H. Lee, X-ray absorption spectroscopy of graphite oxide, EPL 82 (2008) 67004. https://doi.org/10.1209/0295$5075 / 82 / 67004$

[40] A. Ganguly, S. Sharma, P. Papakonstantinou, J. Hamilton, Probing the thermal deoxygenation of graphene oxide using high-resolution in situ X-ray-based spectroscopies, J. Phys. Chem. C 115 (2011) 17009-17019. https://doi.org/10.1021/jp203741y

[41] K. Saravanan, G. Jayalakshmi, K. Suresh, B. Sundaravel, B. K. Panigrahi, D. M. Phase, Structural evaluation of reduced graphene oxide in graphene oxide during ion irradiation: Xray absorption spectroscopy and in-situ sheet resistance studies, Appl. Phys. Lett. 112 (2018) 111907. https://doi.org/10.1063/1.5025097

[42] J. Xu, P. Krueger, C. R. Natoli, K. Hayakawa, Z. Wu, K. Hatada, X-ray absorption spectra of graphene and graphene oxide by full-potential multiple scattering calculations with self- 
$\begin{array}{lllllll}\text { consistent charge density, } & \text { Phys. Rev. } & \text { B } 92 & \text { (2015) } & 125408 .\end{array}$ https://doi.org/10.1103/PhysRevB.92.125408

[43] A.V. Krestinin, A.P. Kharitonov, Yu.M. Shul'ga, O.M. Zhigalina, E.I. Knerelman, M. Dubois, M.M. Brzhezinskaya, A.S. Vinogradov, A.B. Preobrazhenskii, G.I. Zvereva, M.B. Kislov, V.M. Martynenko, I.I. Korobov, G.I. Davydova, V.G. Zhigalina, N.A. Kiselev, Production and characterization of fluorinated single-walled carbon nanotubes, Nanotechnol. Rus. 4 (2009) 60-78. https://doi.org/10.1134/S1995078009010078

[44] M. Brzhezinskaya, E.A. Belenkov, V. A. Greshnyakov, G. E. Yalovega, I. O. Bashkin, New aspects in the study of carbon-hydrogen interaction in hydrogenated carbon nanotubes for energy storage applications, J. Alloy. Compd. $792 \quad$ (2019) 713-720. https://doi.org/10.1016/j.jallcom.2019.04.107

[45] A. Lerf, H. He, M. Forster, J. Klinowski, Structure of graphite oxide revisited, J. Phys. Chem. B 102 (1998) 4477-4482. https://doi.org/10.1021/jp9731821

[46] W. Gao, L. B. Alemany, L. Ci, P. M. Ajayan, New insights into the structure and reduction of graphite oxide, Nat. Chem. 1 (2009) 403-408. https://doi.org/10.1038/nchem.281

[47] C.-H. Chuang, S. C. Ray, D. Mazumder, S. Sharma, A. Ganguly, P. Papakonstantinou, J.W. Chiou, H.-M. Tsai, H.-W. Shiu, C.-H. Chen, H.-J. Lin, J. Guo, W.-F. Pong, Chemical modification of graphene oxide by nitrogenation: an X-ray absorption and emission spectroscopy study, Sci. Rep. 7 (2017) 42235. https://doi.org/10.1038/srep42235

[48] K. Haubner, J. Morawski, P. Olk, L. M. Eng, C. Ziegler, B. Adolphi, E. Jaehne, The route to functional graphene oxide, ChemPhysChem. 11 (2010) 2131-2139. https://doi.org/10.1002/cphc.201000132

[49] L.-Y. Meng, S.-J. Park, Preparation and characterization of reduced graphene nanosheets via pre-exfoliation of graphite flakes, Bull. Korean Chem. Soc. 33 (2012) 209. https://doi.org/10.5012/bkcs.2012.33.1.209

[50] M. Koinuma, H. Tateishi, K. Hatakeyama, S. Miyamoto, C. Ogata, A. Funatsu, T. Taniguchi, Y. Matsumoto, Analysis of reduced graphene oxides by X-ray photoelectron spectroscopy and electrochemical capacitance, Chem. Lett. 42 (2013) 924-926. https://doi.org/10.1246/cl.130152

[51] Y. Shen, Y. Wang, Y. Zhou, C. Hai, J. Hu, Y. Zhang, Electrostatic force spectroscopy revealing the degree of reduction of individual graphene oxide sheets, Beilstein J. Nanotechnol. 9 (2018) 1146-1155. doi:10.3762/bjnano.9.106

[52] S. Böttcher, H. Vita, K. Horn, Photon-induced oxidation of graphene/Ir(111) by $\mathrm{SO}_{2}$ adsorption, Surf. Sci. 641 (2015) 305-309. https://doi.org/10.1016/j.susc.2015.02.003 
[53] V.I. Korepanov, D.M. Sedlovets, An asymmetric fitting function for condensed-phase Raman spectroscopy, Analyst. 143 (2018) 2674-2679. https://doi.org/10.1039/C8AN00710A [54] S. Claramunt, A. Varea, D. López-Díaz, M. M. Velázquez, A. Cornet, A. Cirera, The importance of interbands on the interpretation of the Raman spectrum of graphene oxide, J. Phys. Chem. C 119 (2015) 10123-10123. https://doi.org/10.1021/acs.jpcc.5b01590

[55] X. Díez-Betriu, S. Álvarez-García, C. Botas, P. Álvarez, J. Sánchez-Marcos, C. Prieto, R. Menéndez, A. de Andrés, Raman spectroscopy for the study of reduction mechanisms and optimization of conductivity in graphene oxide thin films, J. Mater. Chem. C 1 (2013) 69056912. https://doi.org/10.1039/C3TC31124D

[56] D. López-Díaz, M. López Holgado, J.L. García-Fierro, M.M. Velázquez, Evolution of the Raman spectrum with the chemical composition of graphene oxide, J. Phys. Chem. C 121 (2017) 20489-20497. https://doi.org/10.1021/acs.jpcc.7b06236

[57] A.C. Ferrari, J. Robertson, Resonant Raman spectroscopy of disordered, amorphous, and diamondlike carbon, Phys. Rev. B $64 \quad$ (2001) 075414. https://doi.org/10.1103/PhysRevB.64.075414

[58] A. Sadezky, H. Muckenhuber, H. Grothe, R. Niessner, U. Pöschl, Raman microspectroscopy of soot and related carbonaceous materials: spectral analysis and structural information, Carbon 43 (2005) 1731-1742. https://doi.org/10.1016/j.carbon.2005.02.018

[59] K.N. Kudin, B. Ozbas, H.C. Schniepp, R.K. Prud'homme, I. Aksay, R. Car, Raman spectra of graphite oxide and functionalized graphene sheets, Nano Lett. 8 (2008) 36-41. https://doi.org/10.1021/n1071822y

[60] E. del Corro, M. Taravillo, V. G. Baonza, Stress-dependent correlations for resonant Raman bands in graphite with defects, J. Raman Spectrosc. 45 (2014) 476-480. https://doi.org/10.1002/jrs.4475

[61] S. Kim, H.J. Jung, J.C. Kim, K-S. Lee, S.S. Park, V.P. Dravid, K. He, H.Y. Jeong, Stressdependent correlations for resonant Raman bands in graphite with defects, ACS Nano 12 (2018) 7335-7342. https://doi.org/10.1021/acsnano.8b03806

[62] H.Q. Wei, P. Zhou, Q.Q. Sun, L.H. Wang, Y. Geng, D.W. Zhang, X.B. Wang, The nanoscale resistive memory effect of graphene oxide, in Proceedings of the 2012 IEEE Nanotechnology Materials and Devices Conference (NMDC2012), Waikiki Beach, HI, USA, Oct 16-19, 2012, pp 54-57. 